\section{Radiochemotherapie: Alles in einer Dosis}

\begin{abstract}
Bei verschiedenen Tumoren gibt es einen Trend hin zur hypofraktionierten Radiotherapie. US-Kollegen haben das nun bei Patienten mit fortgeschrittenem Pankreaskarzinom mit Gemcitabin kombiniert.
\end{abstract}

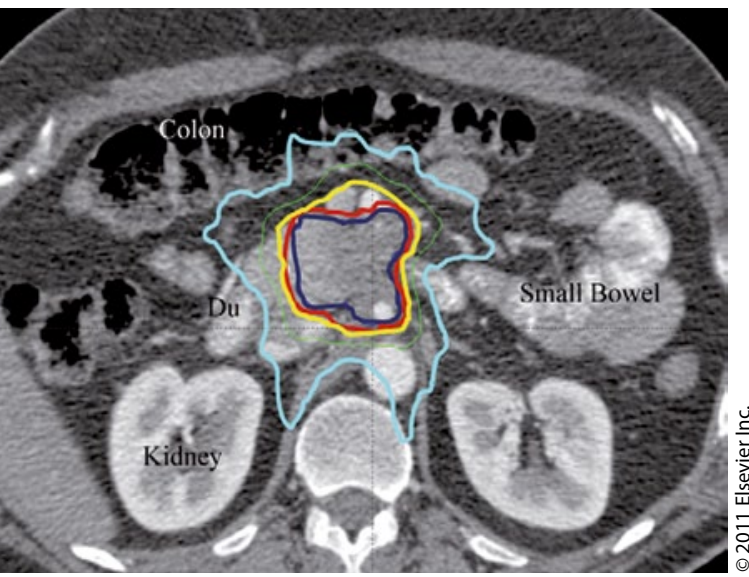

Abb.: Behandlungsplan der stereotaktischen RT (blau: $50 \%$ Iso-Dosis, grün: $75 \%$, gelb: $95 \%$; geplantes Zielvolumen rot konturiert, inneres Zielvolumen dunkelblau)

eim Pankreaskarzinom liegen die Gesamtüberlebensraten nach fünf Jahren bei $5 \%$, inoperable Tumoren gelten als unheilbar. Die in dieser Situation empfohlene multimodale Therapie wurde in jüngerer Zeit immer wieder modifiziert. So zeigte Gemcitabin eine gewisse Wirksamkeit, erstaunlich hohe Ansprechraten wurden jüngst auch in Studien mit hypofraktionierter Bestrahlung (ein bis drei Dosen) erzielt.

In der vorliegenden Phase-II-Studie wurde mit Hilfe eines Linearbeschleunigers eine einzelne Fraktion mittels Körperstereotaxie (SBRT, Abb.) verabreicht, zusätzlich Gemcitabin sequenziell gegeben. 20 Patienten mit lokal fortgeschrittenem, nicht metastasiertem Adenokarzinom erhielten an den Tagen 1, 8 und 15 Gemcitabin, die SBRT mit einer Dosis von 25 Gy an Tag 29. Danach erfolgten drei bis fünf sechswöchige Zyklen Gemcitabin.

Alle Patienten erhielten die SBRT wie geplant und zudem im Median fünf Zyklen Chemotherapie. Zwei Patienten sind noch nach 36,4 bzw. 25,1 Monaten am Leben. Akute nichthämatologische Toxizitäten vom Schweregrad 3 oder höher wurden nicht festgestellt. Späte Toxizitä- ten vom Schweregrad 3 oder höher traten bei einem Patienten auf ( $5 \%)$ : eine Perforation des Duodenums (G4). Drei Patienten (15\%) entwickelten Ulzera (G2), die medikamentös behandelt werden konnten. Insgesamt betrug das mediane Überleben 11,8 Monate; nach einem Jahr lebten noch $50 \%$ der Patienten, nach zwei Jahren $20 \%$. Computertomografien zeigten, dass es nach einem Jahr bei $94 \%$ der überlebenden Patienten nicht zur lokalen Progression gekommen war.

Fazit: Die mit Linearbeschleuniger verabreichte SBRT ergab zusammen mit sequenziellem Gemcitabin eine exzellente lokale Kontrolle des lokal fortgeschrittenen Pankreaskarzinoms. Das klingt vielversprechend, zumal das Auftreten eines lokalen Rezidivs ein signifikanter negativer Prädiktor für das Überleben ist. In einem optimierten Therapieplan mit begleitender Chemotherapie könnte eine hypofraktionierte SBRT das Überleben der Patienten verlängern. Christina Berndt

Schellenberg D et al. Single-fraction stereotactic body radiation therapy and sequential gemcitabine for the treatment of locally advanced pancreatic cancer. Int J Radiat Oncol Biol Phys. 2011;81(1):181-8.

\title{
Operiertes Pankreaskarzinom: wie nachsorgen?
}

Über $80 \%$ der wegen eines Pankreaskarzinoms operierten Patienten erleiden innerhalb von zwei Jahren ein Rezidiv. Bislang gibt es kaum Belege dafür, dass ein frühes Erkennen dieser Rezidive das Leben verlängern kann. Entsprechend uneinheitlich scheint die Nachsorgepraxis.

Z iel der amerikanischen Studie war es, anhand populationsbasierter Daten zu überprüfen, wie Patienten nach einer Resektion in kurativer Absicht nachbeobachtet werden. Bei 2.393 Patienten im Alter von $\geq 66$ Jahren wurden die Nachkontrollen (Arztbesuch und CT) über insgesamt zwei Jahre in vier verschiedenen Zeitfenstern nach der Operation beurteilt. 2.045 Patienten überlebten die ersten drei Monate bis zum Beginn der Kontrollperiode symptomfrei. Insgesamt lag das mediane Überleben bei 14,6 Monaten.

Die Nachsorgepraxis war sehr uneinheitlich. So erhielten etwa im zweiten Beobachtungsjahr $42 \%$ der Überlebenden kein CT, bei $27 \%$ wurden gleich mehrere Aufnahmen gemacht. Insgesamt sank der Anteil der Patienten, bei denen ein CT durchgeführt wurde, von $20,9 \%$ im vierten auf $6,4 \%$ im 27 . Beobachtungsmonat. Feste Intervalle waren nicht erkennbar. Besonders häufig kontrolliert wurden Patienten, die eine adjuvante Chemo- oder Radiotherapie erhalten hatten oder solche, die erst im fortgeschrittenen Alter diagnostiziert worden waren.

In den ersten sechs Monaten nach der Operation waren $52 \%$ der Patienten sowohl bei ihrem Hausarzt als auch bei einem Onkologen in Behandlung. Zwischen dem 22. und 27. postoperativen
Monat nahmen nur $29 \%$ beide Ärzte in Anspruch. Am Ende des Beobachtungszeitraums hatte sich jeder dritte Patient wieder allein seinem Hausarzt anvertraut, obwohl beim Krebsspezialisten deutlich mehr Wert auf bildgebende Verfahren im Rahmen der Nachkontrolle gelegt wurde.

Fazit: Angesichts noch ausstehender evidenzbasierter Leitlinien empfehlen die Autoren, entsprechend den Vorschlägen des National Comprehensive Cancer Network (NCCN), bis zu zwei Jahre postoperativ alle drei bis sechs Monate ein $\mathrm{CT}$ durchzuführen. Prospektive klinische Untersuchungen seien nötig, so die Autoren, um die Effektivität postoperativer Überwachungsstrategien beurteilen zu können.

Christine Starostzik

Sheffield KM et al. Surveillance of pancreatic cancer patients after surgical resection. Ann Surg Oncol. 2011 Dec 6. [Epub ahead of print]. 